\title{
Trap-assisted conductivity in anodic oxide on InSb
}

\author{
G.V. Beketov, A.V. Sukach, V.V. Tetyorkin, S.P. Trotsenko \\ V. Lashkaryov Institute of Semiconductor Physics, NAS of Ukraine, \\ 41, prospect Nauky, 03680 Kyiv, Ukraine \\ E-mail: teterkin@isp.kiev.ua
}

\begin{abstract}
The direct current conductivity of anodic oxide of InSb has been investigated as a function of applied bias and temperature. Proposed in this work is a model of conductivity that includes ohmic, trap-assisted tunneling and Poole-Frenkel conduction processes. Two defect states were found in the energy gap of the anodic oxide, which can be attributed to bulk traps. The asymmetry in the current-voltage characteristics is analyzed in terms of comparative distribution of the applied bias voltage between the anodic oxide and the depletion region in $\mathrm{InSb}$.
\end{abstract}

Keywords: InSb, anodic oxide, traps, conductivity.

Manuscript received 05.10.17; revised version received 08.11.17; accepted for publication 07.12.17; published online 07.12.17.

\section{Introduction}

Narrow-gap InSb is one of the key semiconductor materials that is widely used to manufacture modern infrared detectors [1]. Since InSb is characterized by relatively low melting point, deviation from stoichiometry at the surface as a result of evaporation or segregation of volatile component $(\mathrm{Sb})$ can occur at rather low temperatures. Therefore the surface passivation and protection is recognized to be an important step in technology of InSb infrared detectors. The passivation layer deposited on the surface of infrared photodiodes should provide high resistance, low interface state density and low fixed charge density as well as satisfy requirements for both $n$-type and $p$-type conductivity surface simultaneously. As was first shown by Dewald [2], anodic oxides, grown by the electrolyte technique, can be used as effective passivation layers for InSb photodiodes. Since that, numerous studies of InSb anodic oxides have been published in the literature (see, e.g., [2-10] and citations therein). It was shown that the most important feature of InSb anodization is the composition inhomogeneity of anodic oxides, which strongly depends on the growth conditions. Analysis of published data indicates that physical properties of anodic oxides on $\mathrm{InSb}$ are dependent on a number of factors such as quality and crystallographic orientation of initial wafers, surface treatment before anodization, parameters of anodization process, thermal treatment of oxides after anodization, etc. Because of anodic oxides on InSb have poor chemical and mechanical stability, the passivation stacks comprised the anodic oxide and extrinsic dielectric are usually used. For this purpose, thin layers of $\mathrm{SiO}_{\mathrm{x}}$ and $\mathrm{Si}_{3} \mathrm{~N}_{4}$ were deposited on the anodic oxide by different low-temperature physical and chemical vapor deposition techniques [6-8]. The density of interface states in the range $10^{11} \ldots 10^{12} \mathrm{~cm}^{-2} \cdot \mathrm{eV}^{-1}$ was shown to be typical for the anodic oxide on InSb. Lower densities were obtained when the thermally grown native oxide on the substrate surface was preserved before anodization $[6,9]$. The anodic sulfidization process also provides rather low density interface states of the order of $3 \cdot 10^{10} \mathrm{~cm}^{-2} \cdot \mathrm{eV}^{-1}[10]$.

The commonly used approach for investigation of the anodic oxide on $\mathrm{InSb}$ is based on preparation of MIS-type capacitors. This allows one to use well developed experimental techniques for characterization of anodic oxides themselves as well as their interface with a semiconductor [11]. The direct current conductivity in MIS capacitors of InSb was studied in a number 
of works [12-16]. Because of contradictory data were obtained, studies of electrical conductivity remain important in order to clarify the role of native defects in the anodic oxide. The aim of this work was to study conductivity mechanisms and aging effects in MIS capacitors of InSb.

\section{Samples and experimental methods}

The MIS capacitors were prepared on $n$-InSb(100) substrates with electron concentration in the range $(1 \ldots 2) \cdot 10^{14} \mathrm{~cm}^{-3}$ at the temperature $77 \mathrm{~K}$. Before anodization, all substrates were mechanically and chemomechanically polished on a soft pad wetted with $0.5 \%$ $\mathrm{Br}_{2}$ in methanol. Finally, they were immersed into $\mathrm{Br}_{2}$ in methanol solution for several minutes. In this way, a shiny mirror-like surface was obtained. The anodic oxide was grown using an electrolyte of $0.1 \mathrm{~N} \cdot \mathrm{KOH}$ in a mixture of $90 \%$ ethylene glycol and $10 \% \mathrm{H}_{2} \mathrm{O}$. The anodization process was carried out in two steps. At the first step, the oxide was grown in a galvanostatic mode at a current density $0.1 \mathrm{~mA} / \mathrm{cm}^{2}$ until the voltage of $28 \mathrm{~V}$ on the electrochemical cell was reached. At the second stage, potentiostatic anodization was continued at $28 \mathrm{~V}$ until anodic current drops down to $\sim 10 \mu \mathrm{A} / \mathrm{cm}^{2}$. As measured by ellipsometry, the thickness of the grown oxide was about $450 \mathrm{~nm}$. Indium electrodes with a diameter of $0.75 \mathrm{~mm}$ were deposited on the oxide surface by vacuum evaporation.

In order to investigate aging effects, the capacitors were thermocycled between $77 \mathrm{~K}$ and room temperature for several (typically 5-7) complete cycles. Each thermocycle included fast cooling down to $77 \mathrm{~K}$ and slow warming to room temperature. The conductivity was investigated by measuring the direct current flowing through the anodic oxide as a function of the applied bias and temperature within the range $77 \ldots 300 \mathrm{~K}$.

\section{Results and discussion}

Three groups of MIS capacitors were investigated: initial, subjected to thermal cycling and storaged after cycling for one-month period. The current-voltage characteristics of representative capacitors from each group are shown in Fig. 1. The voltage is assumed to be positive, when the indium electrode on the anodic oxide is positively biased with respect to InSb substrate. The following features of the measured characteristics should be pointed out. The thermal cycling results in increase of the anodic oxide conductivity, that is decreased to the initial state after one-month storage in the laboratory conditions. The measured current is polarity dependent (Fig. 1). At low biases, the current-voltage characteristics exhibit ohmic behavior, whereas at higher biases the nonlinear behavior is observed. Note that the maximum values of the forward current in Fig. 1 correspond to the breakdown voltages 7,8 and $3 \mathrm{~V}$ for the initial capacitor, after storage and thermal cycling, respectively.

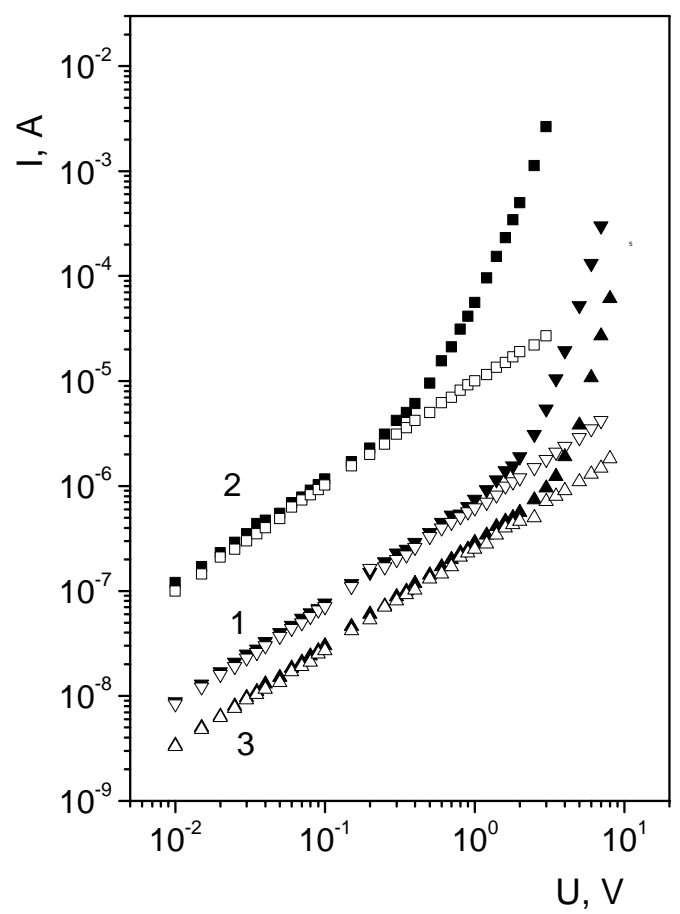

Fig. 1. Reverse (open dots) and forward (close dots) currentvoltage characteristics in initial (1), subjected to thermal cycling (2) and stored for one month (3) InSb MIS capacitors.

The temperature dependences of the leakage current, measured at fixed values of forward and reverse biases, are shown in Fig. 2. As can be seen, there are two temperature intervals where activation dependences of the current on temperature are observed. The activation energies determined from their slope were $E_{1}=0.08 \mathrm{eV}$ and $E_{2}=0.21 \mathrm{eV}$ at low forward and reverse bias voltages, which correspond to the linear $I$ vs $U$ dependences in Fig. 1. These energies decrease to 0.06 and $0.16 \mathrm{eV}$ at the bias voltage $3.0 \mathrm{~V}$, which corresponds to the nonlinear $I$ vs $U$ dependences. After thermal cycling, the corresponding energies were 0.05 and $0.17 \mathrm{eV}$ as well as 0.04 and $0.14 \mathrm{eV}$ at low and high biases, accordingly. The observed activation conductivity can be attributed to thermal ionization of traps in the anodic oxide (hereafter shallow and deep traps). Note that presence of two sets of traps in the anodic oxide of $\mathrm{InSb}$ was previously reported in the literature [5].

As a rule, the polarity dependent current-voltage characteristics of MIS capacitors is interpreted by Schottky emission [11]. This conductivity mechanism was analyzed in details in InSb MIS capacitors [13, 14]. To fit the experimental current-voltage characteristics at room temperature the Schottky current density was calculated using the formula

$$
J_{\mathrm{S}}=A^{*} T^{2}\left(m^{*} / m_{0}\right) \exp \left[-\frac{e}{k T}\left(\Phi_{B}-\sqrt{\frac{e F}{4 \pi \varepsilon_{\infty} \varepsilon_{0}}}\right)\right]
$$




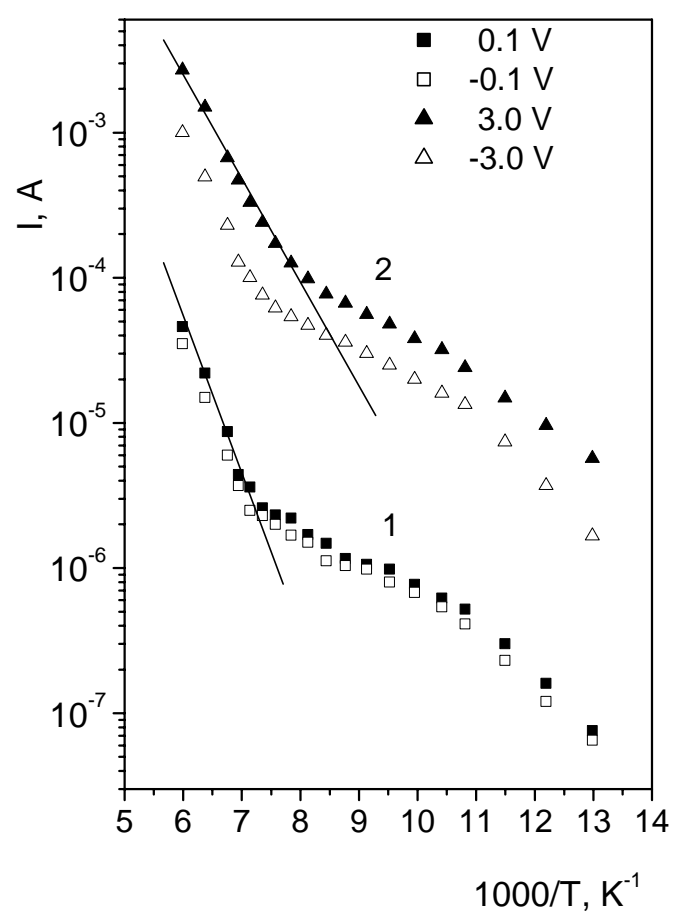

Fig. 2. Temperature dependences of dark current measured before (1) and after (2) thermal cycling. The legend shows the bias voltages in volts.

for the effective mass of electrons in the anodic oxide $m^{*}=0.01 m_{0}$ and the potential barrier height at the interface $\Phi_{B}$ close to $1.0 \mathrm{eV}$ [13]. It should be pointed out that the fitting of calculated data was obtained only at room temperature by using the rather low value of the electron effective mass. In this study, the electron effective mass was estimated in the following way. Because of the anodic oxide is composed of $\operatorname{In}_{2} \mathrm{O}_{3}$ and $\mathrm{Sb}_{2} \mathrm{O}_{3}$, both constituents can contribute to the effective mass. Due to $\mathrm{In}_{2} \mathrm{O}_{3}$ to $\mathrm{Sb}_{2} \mathrm{O}_{3}$ ratio depends on the preparation conditions and remains unknown in the particular sample, the following simplification was made. It was taken into account that $\operatorname{In}_{2} \mathrm{O}_{3}$ constituent prevails in the anodic oxide [6]. Further, it is generally believed that the oxide is amorphous in its nature. However, presence of nanocrystallites in the amorphous phase and formation of microcrystalline structure were observed as well $[5,16]$. So, the values of electron effective mass $m^{*}=0.2 \ldots 0.3 m_{0}$, which was reported in the literature for polycrystalline films and single crystals [18-20], were assumed to be suitable for amorphous $\mathrm{In}_{2} \mathrm{O}_{3}$. These values are typical for electrons in absence of degeneracy. At room temperature, the critical carrier concentration for the onset of degeneracy in $\operatorname{In}_{2} \mathrm{O}_{3}$ is approximately equal to $2 \cdot 10^{18} \mathrm{~cm}^{-3}$ [21]. A similar value of $5.5 \cdot 10^{18} \mathrm{~cm}^{-3}$ was also obtained in [20]. In the presence of degeneracy, the electron effective mass in $\mathrm{In}_{2} \mathrm{O}_{3}$ strongly depends on the electron concentration due to conduction band nonparabolicity.

The results of Schottky emission current calculation (not shown in Fig.1) are restricted to the following reasons. At the temperature $77 \mathrm{~K}$, the calculated values are much smaller as compared to those in Fig. 1, when the barrier height of the order of $1 \mathrm{eV}$ was used. The huge difference between the experimental and calculated values indicates that the asymmetry of $I-$ $U$ characteristics has another nature. In addition, it must be stressed that the Schottky emission may be ineffective in these capacitors with the large thickness of the anodic oxide, because there is the possibility for the injected electrons to be captured to the bulk traps. It is natural to assume that in this case the leakage current is controlled by the Poole-Frenkel emission. In this conductivity mechanism, the charge transfer between localized traps is triggered by field-induced lowering the potential barrier height for the carriers captured in the traps [11]. Obviously, the conductivity process is are hopping in nature and particularly relevant for MIS capacitors with thick oxide, where the relative importance of Schottky emission decreases. The Poole-Frenkel effect is assumed to be applicable for the neutral traps only [22]. Potential barrier lowering occurs as a result of the Coulomb interaction between the released electron and the arising charged trap. For the singly ionized trap, it is given by the following expression:

$$
\Delta \Phi_{\mathrm{PF}}=\beta_{\mathrm{PF}} F^{1 / 2}=\left(\frac{e^{3}}{\pi \varepsilon_{\infty} \varepsilon_{0}}\right)^{1 / 2} F^{1 / 2},
$$

where $F$ is the electric field strength, $\varepsilon_{\infty}$ - optical dielectric constant. Other symbols have their usual meanings. With the lowered barrier, the concentration of thermally activated carriers $n_{0}$ increases yielding the Poole-Frenkel current density [22]

$$
J_{\mathrm{PF}}=e n_{0} \mu F \exp \left(\frac{\Delta \Phi_{\mathrm{PF}}}{k T}\right)
$$

where $n_{0}$ is the thermally equilibrium concentration of electrons, $\mu$-electron mobility. The often cited current density is represented as [11]

$$
J_{\mathrm{PF}} \propto F \exp \left(-\frac{\Phi_{0}-\beta_{\mathrm{PF}} F^{1 / 2}}{k T}\right),
$$

where $\Phi_{0}$ is the energy for the electron transition from the neutral trap to the conduction band. Obviously, experimental $I-U$ curves should be represented by the straight line in the coordinates $\ln \left(J_{\mathrm{PF}} / F\right)-F^{1 / 2}$, if this mechanism is dominant. The slope of the straight line $\beta_{\mathrm{PF}}$ is usually used for estimation of the optical dielectric constant $\varepsilon_{\infty}$. In the case of weak compensation of the donor-like traps the current density is given by

$$
J_{\mathrm{PF}}=e\left(N_{c} N_{t}\right)^{1 / 2} \mu F \exp \left(-\frac{\Phi_{0}-\beta_{\mathrm{PF}} F^{1 / 2}}{2 k T}\right)
$$


where $N_{t}$ is the density of traps, $N_{c}$ - effective density of states in the conduction band [23]. The current-voltage characteristics calculated using the formula (3) are shown in Fig. 3. The calculated curves were obtained for the mean value of the electron mobility $\mu=7 \mathrm{~cm}^{2} / \mathrm{V} \cdot \mathrm{s}$ reported for amorphous $\operatorname{In}_{2} \mathrm{O}_{3}$ films [18-20] and the optical dielectric constant $\varepsilon_{\infty}=14$. The electron concentration $n_{0}$ served as an adjusting parameter.

The Poole-Frenkel conductivity in the investigated capacitors can be explained as follows. At low electric field, ohmic conductivity occurs due to hopping of thermally excited electrons between the traps. With the bias increase, electrons are injected from the InSb substrate to the conduction band of the oxide through the traps located near the interface. The drift of injected electrons in the conduction band, their capture to the bulk traps and subsequent release from the traps result in the Poole-Frenkel conductivity. The injected electrons can be captured by shallow and deep traps, but shallow traps seem to be more effective in the charge transfer at low temperature. However, when the capacitor is heated from $77 \mathrm{~K}$ to room temperature empting of deep traps occurs. Being excited to the conduction band, electrons have large probability to be captured by shallow traps and surface defects. This results in the conductivity increase as well as the shift of $C-U$ curve in Fig. 4 towards the positive bias voltage. In the process of prolong storage at room temperature, the initial distribution of electrons between the deep and shallow traps is restored. In general terms, the proposed model of conductivity correlates with those previously discussed in the literature [5, 24, 25].

To explain the polarity dependent current-voltage characteristics in Fig. 1, one should point out that the investigated capacitors are characterized by rather low resistance of the anodic oxide. Due to the fact that the negatively charged interface results in formation of the depletion region in $\mathrm{InSb}$, the resistance of the substrate and anodic oxide may be comparable. In the forward biased capacitors, the resistance of the depletion region decreases and the applied bias is mainly dropped on the anodic oxide yielding the nonlinear $I-U$ characteristics. At the reverse bias, smaller portion of the applied bias drops on the oxide, which results in the linear (ohmic) $I-$ $U$ characteristics.

Another reason for the asymmetry of the currentvoltage characteristic may be as follows. The composition inhomogeneity in the anodic oxide can cause the electrical inhomogeneity due to formation of graded band gap and non-uniform distribution of charged defects. Since $\mathrm{Ga}_{2} \mathrm{O}_{3}$ has the larger band gap in comparison with $\mathrm{In}_{2} \mathrm{O}_{3}$ [26-28], built-in electric field can appear in the anodic oxide. The presence of this built-in electric field can explain the spontaneous motion of carriers injected from the interface into the anodic oxide at a distance comparable to the oxide layer thickness [25]. To clarify the situation, additional investigations are needed.

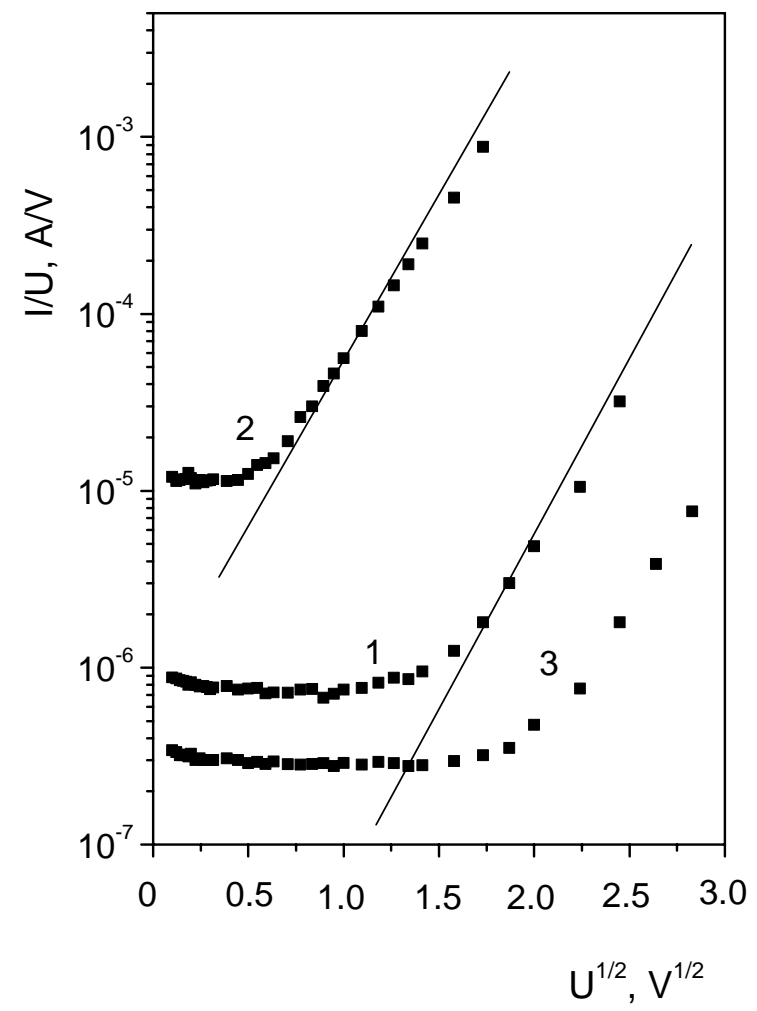

Fig. 3. Measured (dots) and calculated (lines) current-voltage characteristics in initial (1), subjected to thermal cycling (2) and stored for one month (3) InSb MIS capacitors in the Poole-Frenkel coordinates. Parameters for calculation: $\varepsilon_{\infty}=14$, $\mu=7 \mathrm{~cm}^{2} / \mathrm{V} \cdot \mathrm{s}, n_{0}=3 \cdot 10^{6} \mathrm{~cm}^{-3}$ (1) and $5 \cdot 10^{9} \mathrm{~cm}^{-3}$ (2).

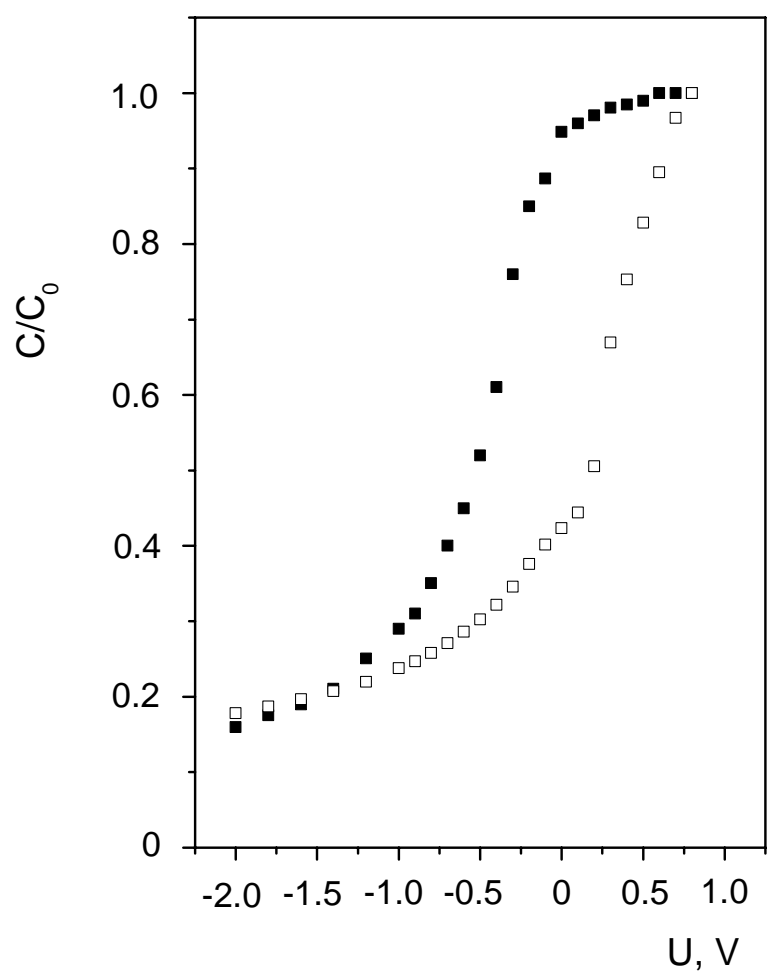

Fig. 4. High-frequency, $1 \mathrm{MHz}$, capacitance-voltage characteristic in MIS capacitor before (close dots) and after (open dots) multiple thermal cycling. 


\section{Conclusions}

1. The current-voltage characteristics in MIS capacitors of $\mathrm{InSb}$ are shown to be polarity dependent. The forward current versus voltage dependences comprise the linear and nonlinear parts, whereas the reverse current linearly depends on the applied bias.

2. Asymmetry and nonlinear behavior of the current-voltage characteristics are explained by comparative contribution of the anodic oxide and the substrate of $n$-InSb to the total resistance of MIS capacitors. The depletion region is caused by the negative fixed charge localized in the anodic oxide and interface.

3. Comparing the measured and calculated current-voltage characteristics, better coincidence was obtained for the Poole-Frenkel emission mechanism of direct current conductivity in the anodic oxide.

\section{References}

1. Rogalski A. Infrared Photon Detectors. SPIE Optical Engineering Press, N.Y., 1995.

2. Dewald J.F. The kinetics and mechanism of formation of anode films on single crystals InSb. $J$. Electrochem. Soc. 1957. 104, No. 4. P. 244-251.

3. Physics and Chemistry of III-V Compound Semiconductor Interfaces, C.W. Wilmsen, Ed. New-York: Plenum, 1985.

4. Chang L.L. and Howard W.E. Surface inversion and accumulation of anodized InSb. Appl. Phys. Lett. 1965. 7, No. 8. P. 210-212.

5. Hung R.Y. and Yon E.T. Surface study of anodized indium antimonide. J. Appl. Phys. 1970. 41, No. 5. P. 2185-2189.

6. Langan I.D., Vismanasan C.R. Characterization of improved InSb interface. J. Vac. Sci. Technol. 1979. 16, No. 5. P. 1474-1477.

7. Kai-Feng Huang, Shie J.S., Luo J.J., and Chen J.S. Electrical properties of InSb metal-insulatorsemiconductor diodes prepared by photochemical vapour deposition. Thin Solid Films. 1987. 151. P. 145-152.

8. Bloom I. and Nemirovsky Y. Surface passivation of back-side illuminated indium antimonide focal plane array. IEEE Trans. Electron Dev. 1993. 40, No. 2. P. 309-314.

9. Barth W. and Lile D. Role of native oxide on indium antimonide surface properties. Thin Solid Films. 1993. 229. P. 54-57.

10. Sun Weiguo. Interface of anodic sulfide on $n$-type InSb. Appl. Phys. A. 1991. 52, No. 1. P. 64-67.

11. Sze S.M. Physics of Ssemiconductors Devices. Second edition. Wiley, 1981.

12. Toshihiko Sakurai, Toshimaza Suzuki and Yoshio Noguchi. Formation and proprties of anodic oxide films on indium antimonide. Jpn. J. Appl. Phys. 1968. 7, No. 12. P. 1491-1496.

13. Wilmsen C.W., Vasbinder G.C. and Chang Y.K. Electrical conduction through thermal and anodic oxides of InSb. J. Vac. Sci. Technol. 1975. 12, No. 1. P. 56-59.

14. Wilmsen C.W. Correlation between the composition profile and electrical conductivity of the thermal and anodic oxides. J. Vac. Sci. Technol. 1976. 13, No. 1. P. 64-67.

15. Sazonov S.G., Yuryev Yu.N. Conductivity of nature oxides on the surface of $\mathrm{A}^{\mathrm{III}} \mathrm{B}^{\mathrm{V}}$ compounds. Optoelectron., Instrument. and Data Process. 1988. No. 3. P. 40-48.

16. Santinacci L., Sproule G.I., Moisa S. et al. Growth and characterization of thin anodic oxide on $n$ $\mathrm{InSb}(100)$ formed in aqueous solutions. Corrosion Sci. 2004. 46. P. 2067-2079.

17. Tang X., van Welzenis R.G., van Setten F.M. and Bosch A.J. Oxidation of the InSb surface at room temperature. Semicond. Sci. Technol. 1986. 1, No. 6. P. 355-365.

18. Scherg-Kurmes H., Seeger S., K rner S. et al. Optimization of the post-deposition annealing process of high-mobility $\mathrm{In}_{2} \mathrm{O}_{3}: \mathrm{H}$ for photovoltaic applications. Thin Solid Films. 2016. 599. P. 78 83.

19. de Wit I.H.W., van Unen G. and Lahey M. Electron concentration and mobility in $\operatorname{In}_{2} \mathrm{O}_{3}$. J. Phys. Chem. Solids. 1977. 38. P. 819-824.

20. Preissler N., Bierwagen O., Ramu A.T., Speck J.S. Electrical transport, electrothermal transport, and effective electron mass in single-crystalline $\mathrm{In}_{2} \mathrm{O}_{3}$ films. Phys. Rev. B. 2013. 88. P. 085305.

21. Zhang K.H.L., Egdell R.G., Offi F. et al. Microscopic origin of electron accumulation in $\mathrm{In}_{2} \mathrm{O}_{3}$. Phys. Rev. Lett. 2013. 110. P. 056803.

22. Schroeder H. Poole-Frenkel effect as dominating current mechanism in thin oxide films - An illusion? J. Appl. Phys. 2015. 117. P. 215103.

23. Vollmann W. Poole-Frenkel conduction in insulators of large impurity densities. phys. status solidi (a). 1974. 22. P. 195-203.

24. Yoram Shapira, Bregman J. and Calahorra Z. Origin and effects of interface traps in anodic native oxides on InSb. Appl. Phys. Lett. 1985. 47, No. 5. P. 495-497.

25. Adar R., Bloom I., Nemirovsky Y. Slow trapping measurements in InSb-anodic oxide interface. Solid-State Electron. 1989. 32, No. 2. P. 111-118.

26. Walsh A., DaSilva J.L.F., Su-Huai Wei et al. Nature of the band gap of $\operatorname{In}_{2} \mathrm{O}_{3}$ revealed by firstprinciples calculations and X-ray spectroscopy. Phys. Rev. Lett. 2008. 100. P. 167402.

27. 27. Tigau N., Ciupina V., Prodan G., Rusu G.I., Gheorghies C., Vasile E. The influence of heat treatment on the electrical conductivity of antimony trioxide thin films. J. Optoelectron. Adv. Mater. 2003. 5. P. 907-912.

28. 28. Tigau N., Ciupina V., Prodan G. Structural, optical and electrical properties of $\mathrm{Sb}_{2} \mathrm{O}_{3}$ thin films with different thickness. J. Optoelectron. Adv. Mater. 2006. 8, No. 1. P. 37-42. 